\title{
Measurement of Bad-Smell Distribution Using Sensing Network Composed of Nodes with Gas Detector Tubes and CCD Image Sensors
}

\author{
Takamichi Nakamoto Member (Tokyo Institute of Technology) \\ Yukinobu Tanaka Student Member (Tokyo Institute of Technology) \\ Hong Phuc Ninh Student Member (Tokyo Institute of Technology)
}

Keywords : : gas detector tube, sensor network, bad smell, image sensor

Recently, the deterioration of environment caused by bad smell becomes one of the problems in our daily life. Ministry of the environment of Japan reported that the numbers of the complaints against bad smells were recently increasing. Thus the rapid and low-cost sensing system is required.

We have developed multi-point bad-smell sensing system for monitoring living environment. Although there are a variety of gas sensors, it is difficult to detect ppb-order gas without interference. Our developed system was able to detect methyl mercaptan, hydrogen sulfide, propionaldehyde and toluene with the concentrations down to a few tens of ppb by using a gas detector tube combined with a CCD image sensor. A gas detector tube with color change caused by the chemical reaction between the analyte and the reagent system was implemented into our low cost wireless portable measurement system. This portable system called a sensor node consists of a gas detector tube, one-dimensional CCD image sensor, FIFO (First In First Out) buffer memory, LVDS interface, microcomputer, and wireless LAN module as is illustrated in Fig.1.

In our experiment, the brightness change from that before gas exposure is integrated along the gas detector tube. This integrated value is called discoloration layer area. We can determine the gas concentration using the discoloration speed when the relationship between time and discoloration layer area is measured. The calibration curve should be prepared prior to the actual measurement after discoloration speeds at several concentrations are obtained.

We used six sensor nodes equipped with gas detector tubes (No.92L, Gastec) and collected the data at a host computer through wireless LAN. The gas detector tubes used here are sensitive to toluene and xylene because they are main components of the solvents in the paint factory. The measurement around a

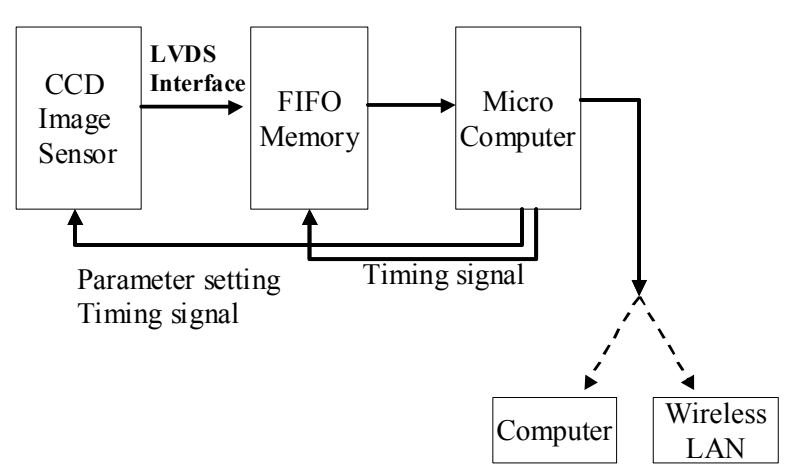

Fig. 1. Block diagram of sensor node paint factory was performed using this sensor network. The sensor node placements around the paint factory are shown in Fig.2. The sensor nodes are placed within the area $(7.8 \mathrm{~m} \times 7 \mathrm{~m})$ around exhaust air duct at the roof.

Fig. 3 shows the result of the xylene gas distribution at a certain time. Sensor1 didn't have response because of its trouble. The sensor 2 responded strongly whereas sensors 3 and 6 seldom responded because these two sensors were away from the plume.

Since the detection limit of xylene of our system was $157 \mathrm{ppb}$, the gas distribution around the exhaust point was clearly obtained. Moreover, the temporal change of the gas concentration during this experiment was observed. Thus, it is possible to measure bad smell distribution using our developed sensor network.

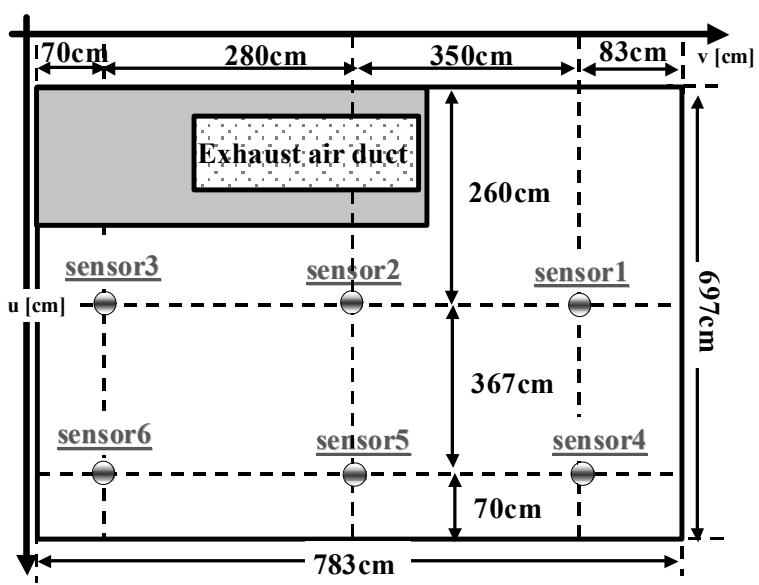

Fig. 2. Sensor node placement around exhaust duct at factory

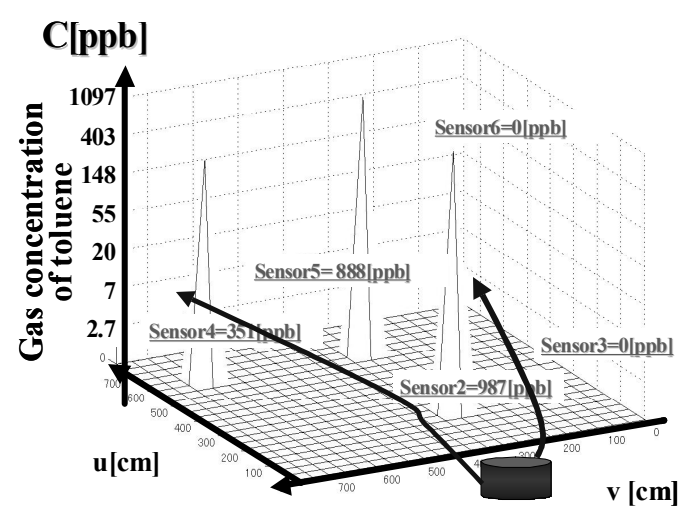

Fig. 3. Xylene gas distribution at roof of paint factory 


\title{
Measurement of Bad-Smell Distribution Using Sensing Network Composed of Nodes with Gas Detector Tubes and CCD Image Sensors
}

\author{
Takamichi Nakamoto* Member \\ Yukinobu Tanaka* Student Member \\ Hong Phuc Ninh* Student Member
}

\begin{abstract}
We have developed multi-point bad-smell sensing system for monitoring living environment. Although there are a variety of gas sensors, it is difficult to detect ppb-order gas without interference. Our previous system was able to detect methyl mercaptan, hydrogen sulfide, propion aldehyde and toluene with the concentrations down to a few tens of ppb by using a gas detector tube combined with a CCD image sensor. A gas detector tube with color change caused by the chemical reaction between the analyte and the reagent system was implemented into our low cost wireless portable measurement system. This portable system called a sensor node consists of a gas detector tube, one-dimensional CCD image sensor, LVDS interface, microcomputer, and wireless LAN module. We report experiment on gas-distribution measurement under actual environments such as paint factories. It was found that the gas distibution around paint factories could be measured using this sensor network. Moreover, the dynamical change of gas distribution was clearly observed.
\end{abstract}

Keywords : gas detector tube, sensor network, bad smell, image sensor

\section{Introduction}

Recently, the deterioration of environment caused by bad smell becomes one of the problems in our daily life. Ministry of the Environment of Japan reported that the numbers of the complaints against bad smells were recently increasing. Thus the rapid and low-cost sensing system is required.

Although the GC/MS (Gas Chromatograph/Mass Spectrometry) method is often used to analyze gases, it is expensive and time-consuming. Moreover, the skill for handling the equipment is required. We focused on gas detector tube to perform bad-smell sensing for monitoring living environment since the gas detector tube technique is well known as a simple method for the gas measurement ${ }^{(1)(2)}$. It is cheap and is easy to handle.

A gas detector tube has not been so far classified into a sensor since it has not been used for continuous measurement. However, we have developed the measurement system to sense the length of color change automatically using an optical scanner ${ }^{(3)(4)}$. Then, a portable sensing system using one-dimensional CCD sensor was developed $^{(5)}$. It is possible to measure a few tens ppb of methyl mercaptan, hydrogen sulfide and propion aldehyde. The sensitivity was much increased than that of the conventional gas detector tube. Moreover, it can be used as a sensor node in a sensor network to obtain gas distribution.

Although preliminary experiment was performed in a wind tunnel $^{(5)}$ and inside a building (corridor) ${ }^{(6)}$, the actual environment around a paint factory is studied in the present study.

Although there have been many reports about a sensor network, few researchers have reported actual chemical sensing network except the preliminary study of hydrogen sensor network ${ }^{(7)}$. This is the first report of bad-smell sensing network.

\footnotetext{
* Graduate school of science and engineering, Tokyo Institute of Technology.

2-12-1, Ookayama, Meguro-ku, Tokyo 152-8552
}

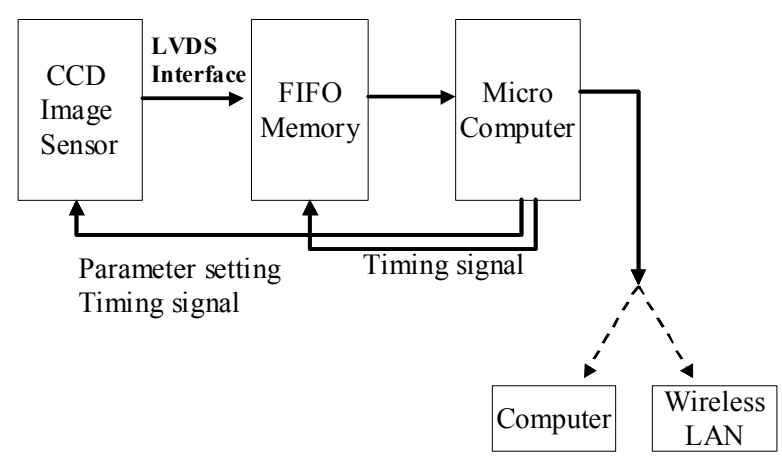

Fig. 1. Block diagram of sensor node.

\section{Experimental Setup}

Although a gas detector tube is manually operated in the conventional method, the image of a gas detector tube is automatically taken and is analyzed. The experimental setup is shown in Fig.1. It consists of one-dimensional CCD image sensor, FIFO (First In First Out) memory and microcomputer. A color CCD image sensor (Toshiba, LIC06A116BH) includes lenses and LEDs for illuminating a gas detector tube. It can cover the length up to $308 \mathrm{~mm}$ enough for the gas detector tube. Although the maximum resolution was 600DPI, the actual resolution used here was 150DPI because of the capacity of FIFO memory. This image sensor outputs 10 bit signals of red, green and blue through LVDS interface.

FIFO memory is required because LVDS signals are too fast for the microcomputer to read. Thus, FIFO memory (IDT72V251, Integrated Device Technology) adopted here has the configuration of $8192 \times 9$ bits and its access time is 10ns enough for LVDS signal (cycle time: 22ns). The data are stored at FIFO memory without the control by a microcomputer. 
The microcomputer (HD64F2633, Renesas) read the image data from FIFO memory at moderate speed. Its clock frequency is $24.576 \mathrm{MHz}$. It also generated several control signals provided to CCD image sensor module. Then, the microcomputer provides the data to a wireless LAN (IEEE802.11b) module (EZL-300W, Alpha project) via RS-232C interface (38.4kBaud). C language was used for programming the microcomputer.

In addition to circuits, a black acrylic box was fabricated to prevent the ambient light from entering the CCD sensor. The distance between the gas detector tube and the CCD sensor was adjusted to obtain the clear image. Before obtaining the image of the gas detector tube, the paper was set below the image sensor for its calibration.

The air pump (N86KN, KF Lab) was used to obtain the flow rate of $90 \mathrm{ml} / \mathrm{min}$. ON/OFF of that pump was controlled by the microcomputer through a power MOS FET (2SK2231) and a relay (G6B-1114P-FD-US DC12, Omron) to realize intermittent measurement. The intermittent measurement allows the system to measure the gas for longer time.

We prepared for six sensor mudules as sensor nodes. A host computer with an access point collects the data from those sensor nodes.

\section{Experiment Inside Wind Tunnel}

Although we have already measured the concentration distribution inside the room (corridor) $^{(6)}$, the intermittent measurement was introduced here. Since the reaction at the gas detector tube is irreversible, it is necessary to prolong the lifetime of the gas detector tube. We have also programmed stopping the pump at rapid increase in concentration.

In our experiment, the discoloration layer area illustrated in Figs.2 (a) and (b) was used. $f_{a}$ in the figure is the brightness average after the color change. The microcomputer calculates the discoloration layer area as the average of 1000 images obtained every $45 \mathrm{~ms}$. The discoloration layer area means the integration of the brightness change from that before gas exposure along the axis of the gas detector tube.

We can determine the concentration using the discolation speed when the relationship between time and the discoloration layer area is measured. The calibration curve should be prepared prior to the actual measurement after discoloration speeds at seveal concentrations are obtained ${ }^{(5)}$. The sensitivity variation from sensor to sensor is shown in the reference ${ }^{(3)}$. This variation is at

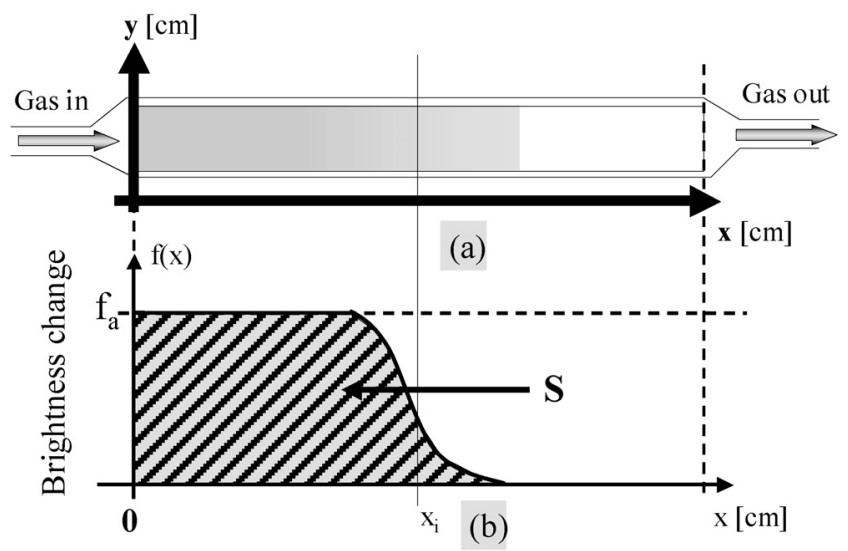

Fig. 2. Discoloration layer area of gas detector tube. (a) brightness, (b) its distribution. most $10 \%$.

The six sensor nodes equipped with gas detector tubes (No.92L, Gastec $^{(8)}$ ) were placed inside the wind tunnel as is shown in Fig.3. The sample used here was propion aldehyde and the liquid sample with the volume of $20 \mathrm{ml}$ was put into the petri dish. The petri dish was placed inside the wind tunnel.

The measurement result is shown in Fig.4 when the average wind speed inside the wind tunnel was $16 \mathrm{~cm} / \mathrm{s}$. It was found that the increase in the discoloration layer area stopped when the pump was OFF. It was also found that the concentration at each sensor

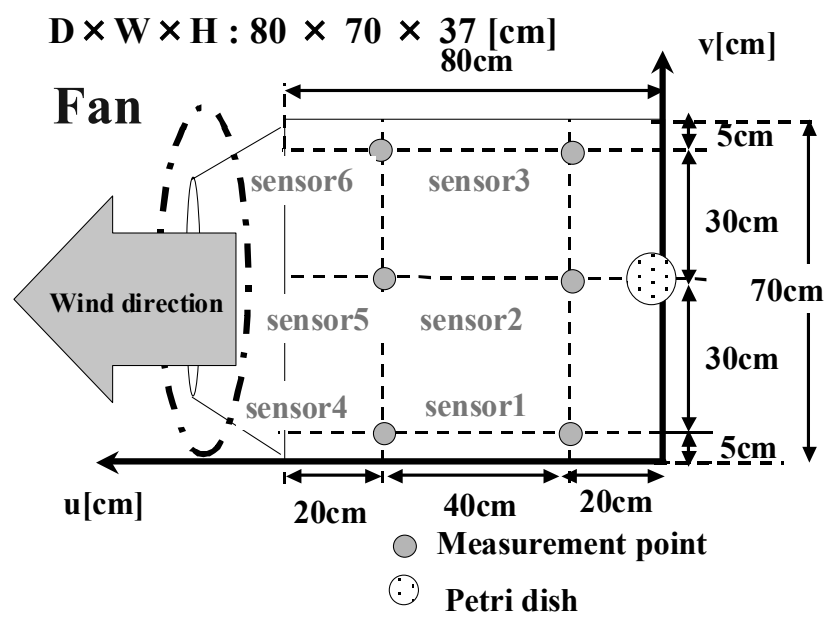

Fig. 3. Sensor nodes inside wind tunnel.

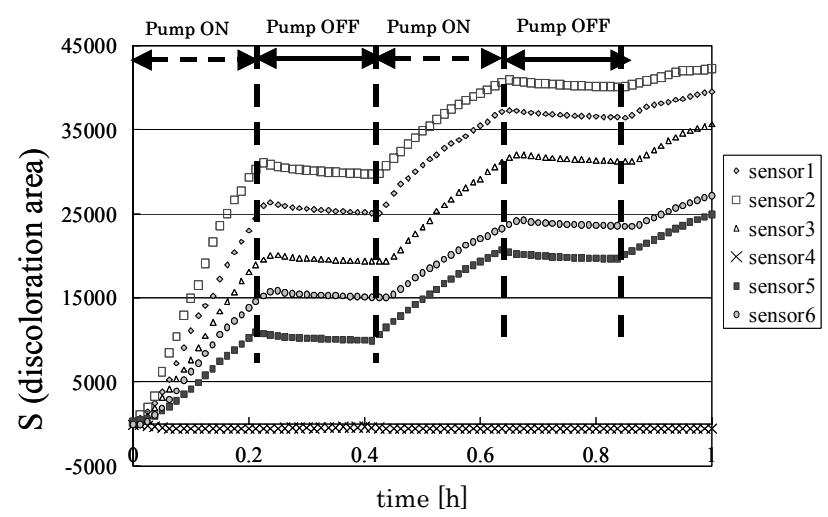

Fig. 4. Measurement result inside wind tunnel.

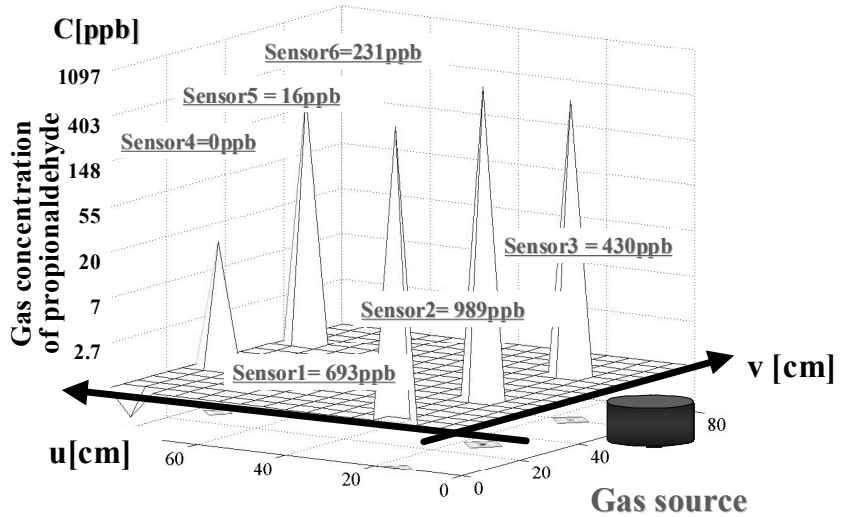

Fig. 5. Obtained gas distribution inside wind tunnel. 
node decreased as time increased. The sensor node 3 seldom responded due to extremely low concentration.

The gas distribution at $9 \mathrm{~min}$ is shown in Fig.5. The concentration at sensor 2 was the largest because it was the closest to the gas source. The gas distribution was not symmetric along the axis of the wind tunnel because of the variation of the wind speed inside the wind tunnel.

\section{Experiment at Paint Factory}

In a paint factory, solvents such as toluene and xylene are often used. Two paint factories gave us the permission of the measurement after looking for appropriate places.

First the relationship between toluene concentration and reaction speed was measured as is shown in Fig.6. The gas packed into a sampling bag was introduced into a gas detector tube (122P, Gastec) in the same procedure described elsewhere ${ }^{(5)}$. The gas concentration was monitored using a photo ionization detector (RAE, PGM-7240). The minimum measured concentration of toluene was $29 \mathrm{ppb}$. The gas detector tube used in this experiment is also sensitive to xylene. Since the actual main component of the solvent was xylene in both factories, the conversion factor was used to obtain xylene concentration. The detection limit of xylene was $157 \mathrm{ppb}$.

In the first factory, we measured xylene concentration around a paint booth inside a building. It was not possible to detect the gas at the factory roof because it has a tall chimney for the exhaust. Thus, we did the measurement inside the building. Sensor node placements around the paint booth are shown in Fig.7.

The sensor nodes Nos.1, 3, 5 had toluene detector tubes and the nodes No.2, 3, 6 had ethyl acetate detector tubes No.141L, Gastec). We only used the data of No.1 and 5 since ethyl acetate detector tubes had the interference with alcohol and the trouble occurred at the node No.3.

The relationship between time and discoloration layer area is shown in Fig. 8. When the variation of discoloration layer area per sampling time is more than 1500 , the pump is stopped to prolong the lifetime of a gas detector tube. One of examples of relationship between time and discoloration layer area is shown in Fig.8. The pump at node 1 was stopped around $0.2 \mathrm{~h}$ and around $0.6 \mathrm{~h}$, and the pump at node 5 was stopped around $0.7 \mathrm{~h}$. The experimental data revealed that the concentration drastically changed around $0.7 \mathrm{~h}$ from $94 \mathrm{ppb}$ to $1954 \mathrm{ppb}$.

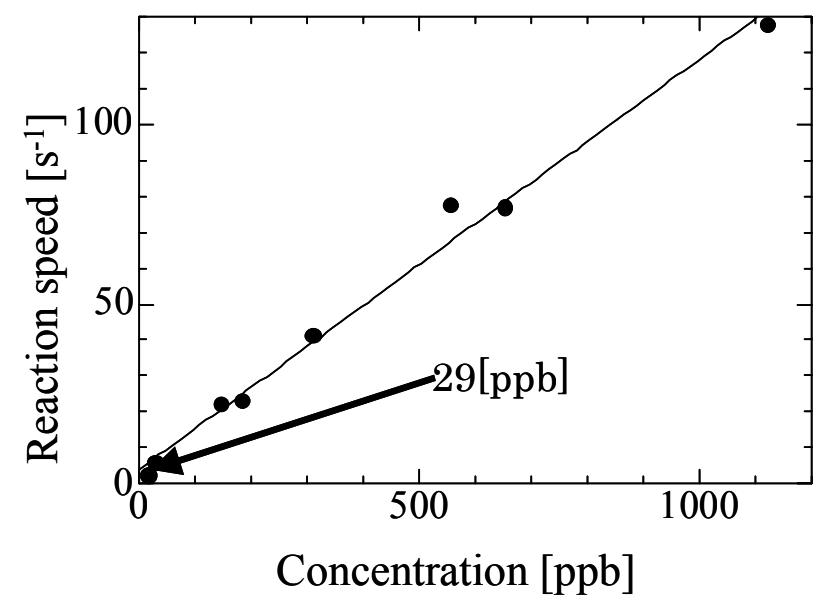

Fig. 6. Relationship between toluene concentration and discoloration speed.
From the data around $0.2 \mathrm{~h}$, the converted xylene concentration at node 1 and node 5 were $90 \mathrm{ppb}$ and $1735 \mathrm{ppb}$ respectively. People feel relatively offensive at the place of node5 and the concentration at node1 was below the level of the offensive smell.

Next, the experiment was performed at the paint factory2. It was possible to measure the gas distribution outside the house in this factory. The sensor node placement at the factory roof is illustrated in Fig.9. The six sensor nodes were placed within the

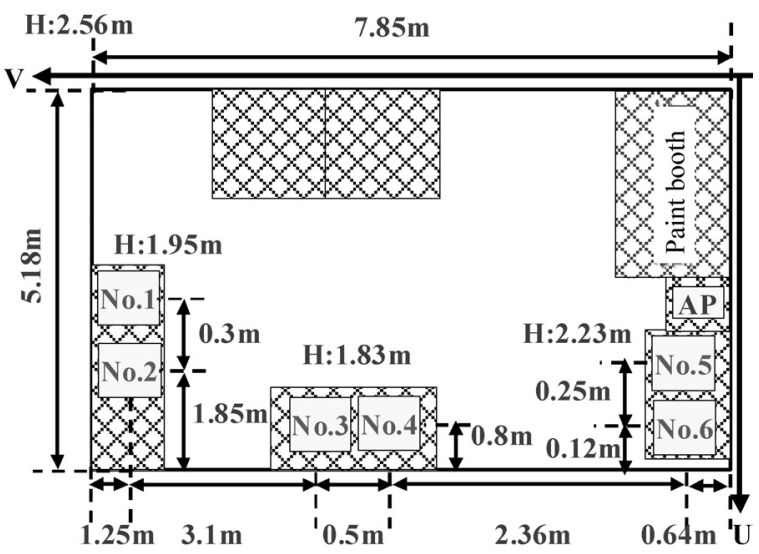

Fig. 7. Sensor node placements around paint booth at factory1.

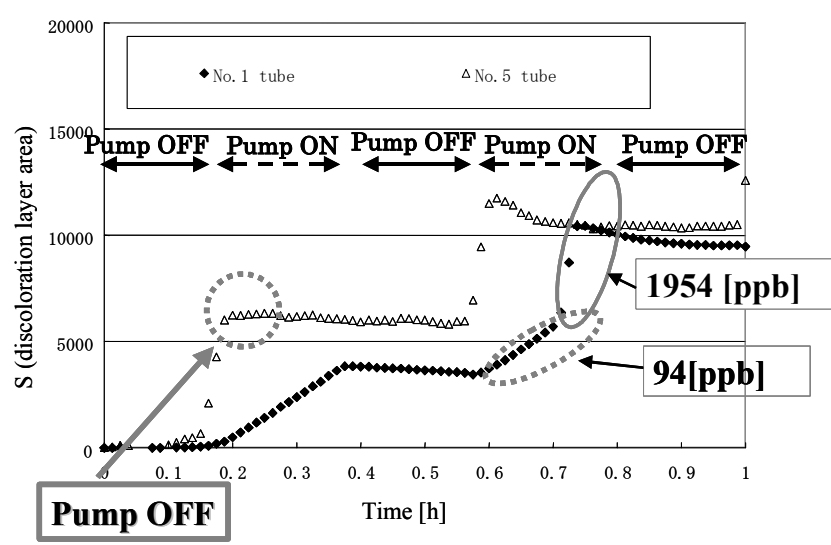

Fig. 8. Relationship between time and discoloration layer area at factory1.

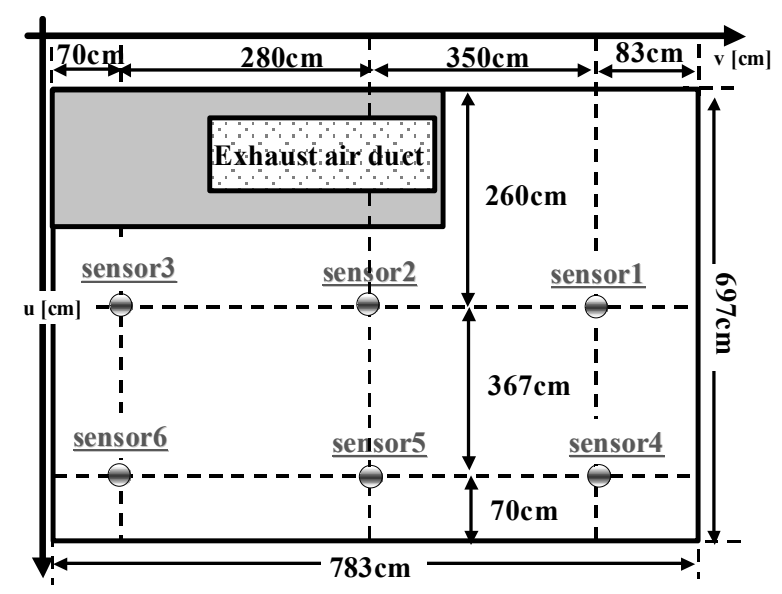

Fig. 9. Sensor node placement around exhaust duct at factory2. 


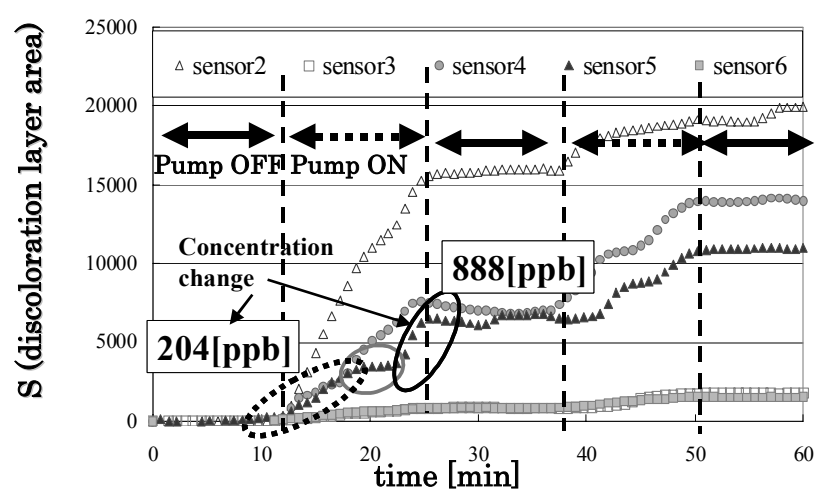

Fig. 10. Relationship between time and disclolation layer area at paint factory 2 .

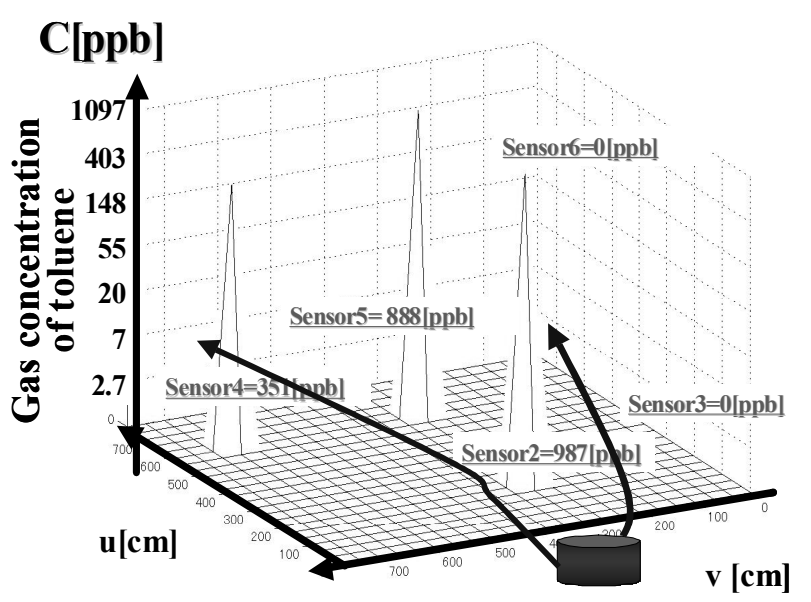

Fig. 11. Gas distribution at roof of factory2.

area $(7.8 \mathrm{~m} \times 7 \mathrm{~m})$ around the exhaust duct. The sensor node 1 was not actually used because of its trouble.

We performed the measurement three times and one of the results is shown in Fig.10. The concentration at sensor node 2 was high and stable since it was the closest to the exhaust air duct. The sensor nodes 3 and 6 seldom responded to gas because they were away from the plume. Although the concentration at sensor node 5 was $204 \mathrm{ppb}$ around $15 \mathrm{~min}$, the concentration increased to $888 \mathrm{ppb}$ around $25 \mathrm{~min}$. The sudden increase in the wind speed often occurs at the roof of the paint factory 2 because it is next to the railway and a train frequently passes at that place. The concentration change was caused both by this sudden wind and the painting operation at the booth. Thus, the dynamical change of gas concentration can be observed using this sensor network..

The gas distribution around 10min is shown in Fig.11. The gas disribution can be clearly observed using our developed sensor network.

Then, the lifetime of a gas detector tube is discussed. In case of the concentration higher than a few ppm, it works for a few hours using the intermittent gas sampling, whereas it only works within $10 \mathrm{~min}$ without the intermittent gas sampling. Moreover, the lifetime of a gas detector tube is longer in case of low concentration. Its characteristic did not change after the ambient air was continuously supplied to a gas detector tube for one day.

\section{Conclusion}

We have developed the sensor network composed of sensor nodes with gas detector tubes and one-dimensional CCD sensors. The intermittent measurement was introduced into the system to prolong lifetime of a gas detector tube. Using that sensor network, xylene distribution around a paint factory was successfully obtained. Moreover, the dynamical change of xylene cocentration was clearly observed. It is possible to monitor gas distribution using that sensor network. Further experiment will be achieved to verify the usefulness of a sensor network composed of sensor nodes with gas detector tubes and CCD image sensors.

\section{Acknoledgements}

We wish to thank Mr.N.Kinoshita of Tokyo Metropolitan Industrial Technology Research Institute for helpful advice. We also wish to thank Mr.K.Shinoda of Sakura Painting Co. and Mr.Y.Hayashi of Ajiadenso Co. for giving us opportunities to do the measurements.

(Manuscript received Nov. 14, 2006, revised Feb. 5, 2007)

\section{References}

(1) Yokogawa Analytical Systems (YAN) Environmental Measurements Technical Committee, Environmental Measurements, Ohmsha, pp.15-47 (2000) (in Japanese)

(2) W. Bather : "Detector tube technology", Sensor Update, 4, p.82-107 (1998)

(3) Y. Tanaka, M. Yoshioka, T. Nakamoto, and T. Moriizumi : "Study of bad-smell sensing system”, IEEJ Tran. SM, 124, p.321 (2004) (in Japanese)

(4) Y. Tanaka, M. Yoshioka, T. Nakamoto, and T. Moriizumi : "A study of gas inference and sensitivity increase in optical bad smell sensing system using gas detector tube", IEEJ Trans. SM, 125, p.64 (2005) (in Japanese)

(5) Y. Tanaka, T. Nakamoto, and T. Moriizumi : "Study of highly sensitive smell sensing system using gas detector tube combined with optical sensor", Sensors and Actuators B, 119, p.84 (2006)

(6) Y. Tanaka and T. Nakamoto : Proc.JSTS, 2005, p.299 (in Japanese)

(7) K. Yokosawa, S. Nakano, Y. Goto, and K. Tsukada : "Prototype system comprising battery-powered sensor nodes and a wireless network for detecting hydrogen leaks", Proc. $22^{\text {nd }}$ Sensor Symp., p.435 (2005)

(8) Environmental analysis technology $5^{\text {th }}$ Edition, GASTEC, p.2-9 (2004)

Takamichi Nakamoto (Member) received the $\mathrm{BE}, \mathrm{ME}$ and $\mathrm{PhD}$ degrees

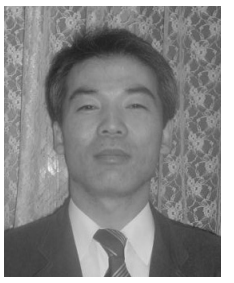
from the Tokyo Institute of Technogy, Tokyo, Japan in 1982, 1984 and 1991 respectively. He was with Hitachi Ltd from 1984 to 1987. In 1987, he joined the Tokyo Institute of Technology as a research associate. He is currently an associate professor with the dempartment of physical electronics, Tokyo Institute of Technology. From 1996 to 1997, he was a visiting researcher at Pacific Northwest Laboratories, Richland WA, USA. His research interests include chemical sensing systems, neural network, ASIC chip design and virtual reality related to olfaction.

Yukinobu Tanaka (Student Member) received the BE degree from the Osaka Institute of Technology, Osaka, Japan in 2000, the ME degree form the Tokyo Institute of Technology, Tokyo, Japan in 2003 and the degree of Dr. Eng. from the same institute in 2006, respectively. He currently works for Hitachi Ltd.

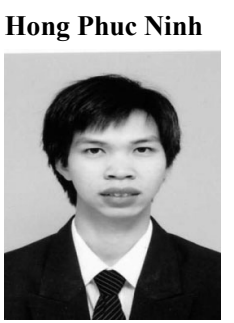

(Student Member) received the BE degree from the Tokyo Institute of Technology, in 2006, where he is currently pursuing the ME degree. 